\title{
Constrained Weak Nash-Type Equilibrium Problems
}

\author{
W. C. Shuai, ${ }^{1,2}$ K. L. Xiang, ${ }^{2}$ and W. Y. Zhang ${ }^{2}$ \\ ${ }^{1}$ Department of Mathematics, Sichuan University for Nationalities, Kangding 626000, China \\ ${ }^{2}$ Department of Economic Mathematics, Southwestern University of Finance and Economics, Chengdu 610000, China
}

Correspondence should be addressed to W. Y. Zhang; zhangwy@swufe.edu.cn

Received 28 January 2014; Accepted 20 March 2014; Published 14 April 2014

Academic Editor: Sheng-Jie Li

Copyright (C) 2014 W. C. Shuai et al. This is an open access article distributed under the Creative Commons Attribution License, which permits unrestricted use, distribution, and reproduction in any medium, provided the original work is properly cited.

\begin{abstract}
A constrained weak Nash-type equilibrium problem with multivalued payoff functions is introduced. By virtue of a nonlinear scalarization function, some existence results are established. The results extend the corresponding one of Fu (2003). In particular, if the payoff functions are singlevalued, our existence theorem extends the main results of Fu (2003) by relaxing the assumption of convexity.
\end{abstract}

\section{Introduction}

For a long time, real valued functions have played a central role in game theory. More recently, motivated by applications to real-world situations, many authors have studied the existence of solutions of Pareto equilibria of multiobjective game with vector payoff functions; for example, see [1-4] and the references therein. Notice that most payoffs may be one collection of things from many collections of things in the real world; reference [5] studied the constrained Nash-type equilibrium problem with multivalued payoff functions and proved the existence results.

In the paper, let $I$ be an index set, $Z_{i}$ a real topological vector space, and $X_{i}(i \in I)$ a Hausdorff topological space. Let $X=\prod_{i \in I} X_{i}$ and $X^{i}=\prod_{j \in I, j \neq i} X_{j}$. For each $x \in X$, let $x_{i}$ and $x^{i}$ denote the $i$ th coordinate of $x$ and the projection of $x$ on $X^{i}$, respectively. In the sequel, we may write $x=\left(x_{i}\right)_{i \in I}=\left(x_{i}, x^{i}\right)$. For all $i \in I$, let $C_{i}$ be a convex, closed, and pointed cone of $Z_{i}$, with apex at the origin and with nonempty interior; let $F_{i}: X_{i} \times X^{i} \rightarrow 2^{Z_{i}}$ and $S_{i}: X \rightarrow 2^{X_{i}}$. We consider a class of constrained weak Nash-type equilibrium problems with multivalued payoff functions.

(CWNEP) Finding an $\bar{x}=(\bar{x})_{i \in I} \in X$ such that, for each $i \in I, u_{i} \in S_{i}(\bar{x})$, and $\bar{z}_{i} \in F_{i}\left(\bar{x}_{i}, \bar{x}^{i}\right)$, there exists $z_{i} \in F_{i}\left(u_{i}, \bar{x}^{i}\right)$ satisfying

$$
z_{i}-\bar{z}_{i} \notin-\operatorname{int} C_{i} .
$$

Then, $\bar{x}$ is a solution of $(C W N E P)$.
The following problems are special cases of (CWNEP).

(i) If, for each $i \in I, F_{i}$ is a singlevalued function, $Z_{i}=$ $R$, and $S_{i}(X)=X_{i},(C W N E P)$ reduces to the Nash equilibrium problem [6].

(ii) Let $X, Y$, and $Z$ be real Hausdorff topological vector spaces, and let $C$ and $D$ be two nonempty subsets of $X$ and $Y$, respectively. Let $P \subset Z$ be a closed convex and pointed cone with int $P \neq \emptyset$, let $S: C \times D \rightarrow 2^{C}$ and $T: C \times D \rightarrow 2^{D}$ be two set-valued mappings, and let $f, g: C \times D \rightarrow Z$ be two vector-valued mappings. The problem (CWNEP) reduces to a class of symmetric vector quasiequilibrium problems (for short, SVQEP) that consists in finding $(\bar{x}, \bar{y}) \in C \times D$ such that $\bar{x} \in S(\bar{x}, \bar{y}), \bar{y} \in T(\bar{x}, \bar{y})$, and

$$
\begin{aligned}
& f(x, \bar{y})-f(\bar{x}, \bar{y}) \notin-\operatorname{int} P, \quad \forall x \in S(\bar{x}, \bar{y}), \\
& g(\bar{x}, y)-g(\bar{x}, \bar{y}) \notin-\operatorname{int} P, \quad \forall y \in T(\bar{x}, \bar{y}),
\end{aligned}
$$

which was considered by $\mathrm{Fu}[7]$.

In this paper, we obtain the existence result for (CWNEP). Our existence theorem extends the main result of [6] from singlevalued case to multivalued case. In particular, if the payoff functions are singlevalued, our existence theorem extends the corresponding result in [7] by relaxing the assumption of convexity. 
The rest of the paper is organized as follows. In Section 2, we state some notations and preliminary results for multivalued mappings. We recall the nonlinear scalarization function and its properties. In Section 3, we show existence result for (CWNEP).

\section{Preliminaries}

Let us first recall some definitions of continuity for setvalued mappings. Let $X$ and $Y$ be two topological spaces. $T: X \rightarrow 2^{Y}$ is a set-valued mapping. $T$ is said to be upper semicontinuous at $x_{0} \in X$ if, for each open set $V$ containing $T\left(x_{0}\right)$, there is an open set $U$ containing $x_{0}$ such that, for each $t \in U, T(t) \subseteq V$. It is said to be upper semicontinuous if it is upper semicontinuous at every point $x \in X$. T is said to be lower semicontinuous at $x_{0} \in X$ if, for each open set $V$ with $T\left(x_{0}\right) \cap V \neq \emptyset$, there is an open set $U$ containing $x_{0}$ such that, for each $t \in U, T(t) \cap V \neq \emptyset$. It is said to be lower semicontinuous on $X$ if it is lower semicontinuous at every point $x \in X$.T is said to be continuous at $x_{0}$ if it is both upper semicontinuous and lower semicontinuous at $x_{0}$. It is said to be continuous on $X$ if it is continuous at every point $x \in X$.

From [7, Lemma 2], $T$ is 1.s.c. at $x \in X$ if and only if, for any $y \in T(x)$ and any net $\left\{x_{n}\right\}, x_{n} \rightarrow x$, there is a net $\left\{y_{n}\right\}$ such that $y_{n} \in T\left(x_{n}\right)$ and $y_{n} \rightarrow y . T$ is closed if and only if, for any net $\left\{x_{n}\right\}, x_{n} \rightarrow x$, and any net $\left\{y_{n}\right\}, y_{n} \in T\left(x_{n}\right)$, $y_{n} \rightarrow y$, one has $y \in T(x)$.

Definition 1. Assume that $X$ is a Hausdorff topological space and $Z$ is a real topological vector space. Let $E$ be a nonempty convex subset of $X$, let $H: E \rightarrow 2^{Z}$ be a set-valued mapping, and let $P \subset Z$ be a closed convex and pointed cone with int $P \neq \emptyset$. $H$ is said to be generalized Luc's quasi$P$-convex on $E$ if, for every $x_{1}, x_{2} \in E, \lambda \in[0,1]$, and $y \in H\left(\lambda x_{1}+(1-\lambda) x_{2}\right)$, there exist $z_{1} \in H\left(x_{1}\right)$ and $z_{2} \in H\left(x_{2}\right)$ such that

$$
y \in z-C, \quad z \in C\left(z_{1}, z_{2}\right)
$$

where $C\left(z_{1}, z_{2}\right)$ is the set of all upper bounds of $z_{1}$ and $z_{2}$; that is,

$$
C\left(z_{1}, z_{2}\right)=\left\{z \in Z \mid z_{1} \in z-P, z_{2} \in z-P\right\} .
$$

Remark 2. Definition 1 is a generalization of the concept of Luc's quasi- $P$-convexity in [8].

Now we recall the definition of the nonlinear scalarization function $[9,10]$ as follows.

Definition 3. Let $Z$ be a real topological vector space, and let $P \subset Z$ be a closed convex and pointed cone with $e \in$ int $P$. The nonlinear scalarization function $\xi_{e}: Z \rightarrow R$ is defined by

$$
\xi_{e}(y)=\min \{t \in R \mid y \in t e-P\} .
$$

Lemma 4 (see [9]). The nonlinear scalarization function has the following main properties:

(i) $\xi_{e}(\cdot)$ is continuous and convex; (ii) $\xi_{e}(\cdot)$ is subadditive; that is, $\xi_{e}\left(y_{1}+y_{2}\right) \leq \xi_{e}\left(y_{1}\right)+\xi_{e}\left(y_{2}\right)$;

(iii) $\xi_{e}(\cdot)$ is strictly monotone; that is, if $y_{1}-y_{2} \in$ int $P$, then $\xi_{e}\left(y_{1}\right)>\xi_{e}\left(y_{2}\right)$.

\section{Existence for the Solution of (CWNEP)}

Throughout this section, let $E_{i}(i \in I)$ be a locally convex Hausdorff topological vector space, and let $Z_{i}$ be a real Hausdorff topological vector space. Let $X_{i}$ be a nonempty, compact convex subset of $Z_{i}$, respectively. Let $C_{i} \subset Z_{i}$ be a closed convex and pointed cone with $e_{i} \in \operatorname{int} C_{i}$. Suppose that $S_{i}: X \rightarrow 2^{X_{i}}$ is a continuous set-valued mapping with compact convex values and $F_{i}: X_{i} \times X^{i} \rightarrow 2^{Z_{i}}$ is a continuous set-valued mapping with compact values. For every $i \in I$, set $\xi_{e_{i}}\left(F_{i}(x, y)\right)=\bigcup_{u_{i} \in F_{i}(x, y)} \xi_{e_{i}}\left(u_{i}\right)$.

Lemma 5 (see [11]). Let $E$ be a nonempty compact convex subset of a locally convex Hausdorff topological space $X$. If $G: E \rightarrow 2^{E}$ is upper semicontinuous and, for each $x \in E$, $G(x)$ is a nonempty, closed, and convex subset, then there exists an $\bar{x} \in E$ such that $\bar{x} \in G(\bar{x})$.

Theorem 6. Suppose that the following conditions hold:

(i) $S_{i}: X \rightarrow 2^{X_{i}}$ is continuous with compact convex values;

(ii) $F_{i}: X_{i} \times X^{i} \rightarrow 2^{Z_{i}}$ are continuous with compact values;

(iii) for each fixed $x_{i} \in X_{i}, F_{i}\left(\cdot, x^{i}\right)$ is generalized Luc's quasi- $C_{i}$-convex.

Then, there exists an $\bar{x} \in X_{i} \times X^{i}$ such that, for each $i \in I, u_{i} \in$ $S_{i}(\bar{x})$, and $\bar{z}_{i} \in F_{i}\left(\bar{x}_{i}, \bar{x}^{i}\right)$, there exists $z_{i} \in F_{i}\left(u_{i}, \bar{x}^{i}\right)$ satisfying

$$
z_{i}-\bar{z}_{i} \notin-\operatorname{int} C_{i}
$$

Proof. We define a set-valued mapping $A_{i}: X \rightarrow 2^{X_{i}}$ by

$$
\begin{aligned}
A_{i}(x)=\left\{u_{i} \in S_{i}(x) \mid \max \xi_{e_{i}}\left(F_{i}\left(u_{i}, x^{i}\right)\right)\right. \\
\left.=\min \bigcup_{x_{i} \in S_{i}(x)} \max \xi_{e_{i}}\left(F_{i}\left(x_{i}, x^{i}\right)\right)\right\} .
\end{aligned}
$$

It follows from [12, pages 110-119, Propositions 6 and 21] that $\max \xi_{e_{i}}\left(F_{i}\left(\cdot, x^{i}\right)\right)$ is upper semicontinuous for each fixed $x^{i} \in X^{i}$. By [12, page 112, Proposition 11], the set

$$
\bigcup_{\theta \in S(x)} \max \xi_{e_{i}}(F(\theta, y))
$$

is compact. Therefore, $A_{i}(x)$ is nonempty for every $x \in X$.

Let

$$
\begin{gathered}
\left\{x_{n}\right\} \in X, \quad x_{n} \longrightarrow x_{0}, \\
u_{i, n} \in A_{i}\left(x_{n}\right), \quad u_{i, n} \longrightarrow u_{i, 0} .
\end{gathered}
$$


We must show that $u_{i, 0} \in A_{i}\left(x_{0}\right)$. First, note that $u_{i, n} \in A_{i}\left(x_{n}\right)$ and then $u_{i, n} \in S_{i}\left(x_{n}\right)$. As $S_{i}(\cdot)$ is upper semicontinuous and the set $S_{i}\left(x_{0}\right)$ is compact, it follows that $u_{i, 0} \in S_{i}\left(x_{0}\right)$. Suppose that $u_{i, 0} \notin A_{i}\left(x_{0}\right)$. Then, there exists a vector $w_{i, 0} \in S_{i}\left(x_{0}\right)$ satisfying

$$
\max \xi_{e_{i}}\left(F_{i}\left(w_{i, 0}, x_{0}^{i}\right)\right)<\max \xi_{e_{i}}\left(F_{i}\left(u_{i, 0}, x_{0}^{i}\right)\right) .
$$

As $S_{i}(\cdot)$ is lower semicontinuous, there exists $w_{i, n} \in S_{i}\left(x_{n}\right)$, such that $w_{i, n} \rightarrow w_{i, 0}$. It follows from compactness of $F_{i}\left(w_{i, n}, x_{n}^{i}\right)$ that there exists $z_{i, n} \in F_{i}\left(w_{i, n}, x_{n}^{i}\right)$ such that

$$
\xi_{e_{i}}\left(z_{i, n}\right)=\max \xi_{e_{i}}\left(F_{i}\left(w_{i, n}, x_{n}^{i}\right)\right) .
$$

It follows from the upper semicontinuity of $F_{i}(\cdot, \cdot)$ and the compactness of $X^{i} \times X_{i}$ that $F_{i}\left(x^{i}, x_{i}\right)$ is compact. Hence, for the net $\left\{z_{i, n}\right\}$, there exists a subnet of $\left\{z_{i, n}\right\}$ converging to $z_{i, 0}$. Without loss of generality, assume $z_{i, n} \rightarrow z_{i, 0}$. Now we prove that

$$
\xi_{e_{i}}\left(z_{i, 0}\right)=\max \xi_{e_{i}}\left(F_{i}\left(w_{i, 0}, x_{0}^{i}\right)\right)
$$

Since the mapping $F_{i}(\cdot, \cdot)$ is upper semicontinuous and the set $F_{i}\left(w_{i, 0}, x_{0}^{i}\right)$ is compact, we have $\xi_{e_{i}}\left(z_{i, 0}\right) \in \xi_{e_{i}}\left(F_{i}\left(w_{i, 0}, x_{0}^{i}\right)\right)$.

Now, suppose that $\xi_{e_{i}}\left(z_{i, 0}\right) \neq \max \xi_{e_{i}}\left(F_{i}\left(w_{i, 0}, x_{0}^{i}\right)\right)$. Namely, there exists $v_{i, 0} \in F_{i}\left(w_{i, 0}, x_{0}^{i}\right)$ such that $\xi_{e_{i}}\left(v_{i, 0}\right)>\xi_{e_{i}}\left(z_{i, 0}\right)$. As $F_{i}(\cdot, \cdot)$ is lower semicontinuous, there exists $v_{i, n} \in F_{i}\left(w_{i, n}, x_{n}^{i}\right)$ such that $v_{i, n} \rightarrow v_{i, 0}$. Since $\xi_{e_{i}}(\cdot)$ is continuous, for $n$ large enough,

$$
\xi_{e_{i}}\left(v_{i, n}\right)>\xi_{e_{i}}\left(z_{i, n}\right),
$$

which is a contradiction to (11).

From the compactness of $F_{i}\left(u_{i, n}, x_{n}^{i}\right)$, we take $\widetilde{z}_{i, n} \in$ $F\left(u_{i, n}, x_{n}^{i}\right)$ such that

$$
\xi_{e_{i}}\left(\tilde{z}_{i, n}\right)=\max \xi_{e_{i}}\left(F_{i}\left(u_{i, n}, x_{n}^{i}\right)\right)
$$

By the compactness of $F_{i}\left(x_{i}, x^{i}\right)$, we can choose a converging subnet of $\left\{\widetilde{z}_{i, n}\right\}$, which is denoted without loss of generality by the original net $\left\{\widetilde{z}_{i, n}\right\}$. Assume $\widetilde{z}_{i, n} \rightarrow \widetilde{z}_{i, 0}$. Similar to the preceding proof, we have

$$
\xi_{e_{i}}\left(\widetilde{z}_{i, 0}\right)=\max \xi_{e_{i}}\left(F\left(u_{i, 0}, x_{0}^{i}\right)\right) .
$$

Then, by $(10), \xi_{e_{i}}\left(z_{i, 0}\right)<\xi_{e_{i}}\left(\widetilde{z}_{i, 0}\right)$.

It follows from the continuity of $\xi_{e_{i}}(\cdot)$ that $\xi_{e_{i}}\left(z_{i, n}\right) \rightarrow$ $\xi_{e_{i}}\left(z_{i, 0}\right)$ and $\xi_{e_{i}}\left(\widetilde{z}_{i, n}\right) \rightarrow \xi_{e_{i}}\left(\widetilde{z}_{i, 0}\right)$. Therefore, $\xi_{e_{i}}\left(z_{i, n}\right)<\xi_{e_{i}}\left(\widetilde{z}_{i, n}\right)$, when $n$ is large enough. It is said that

$$
\max \xi_{e_{i}}\left(F_{i}\left(w_{i, n}, x_{n}^{i}\right)\right)<\max \xi_{e_{i}}\left(F_{i}\left(u_{i, n}, x_{n}^{i}\right)\right) .
$$

By the definition of $A_{i}(\cdot)$ and $u_{i, n} \in A_{i}\left(x_{n}\right)$, we have

$$
\max \xi_{e_{i}}\left(F_{i}\left(u_{i, n}, x_{n}^{i}\right)\right)=\min \bigcup_{x_{i, n} \in S_{i}\left(x_{n}\right)} \max \xi_{e_{i}}\left(F_{i}\left(x_{i, n}, x_{n}^{i}\right)\right) .
$$

This, however, contradicts the fact $u_{i, n} \in A_{i}\left(x_{n}\right)$. Therefore, the mapping $A_{i}(\cdot)$ is closed.

$$
\text { Let } \begin{aligned}
u_{i, 1}, u_{i, 2} \in A_{i}(x), \lambda \in(0,1) \text {, and } \\
\qquad \alpha_{0}=\min \bigcup_{\theta_{i} \in S_{i}(x)} \max \xi_{e_{i}}\left(F_{i}\left(\theta_{i}, x^{i}\right)\right) .
\end{aligned}
$$

From the definition of $A_{i}(\cdot)$, we have $u_{i, 1}, u_{i, 2} \in S_{i}(x)$ and

$$
\max \xi_{e_{i}}\left(F\left(u_{i, 1}, x^{i}\right)\right)=\max \xi_{e_{i}}\left(F\left(u_{i, 2}, x^{i}\right)\right)=\alpha_{0} .
$$

As $S_{i}(x)$ is convex-valued, $\lambda u_{i, 1}+(1-\lambda) u_{i, 2} \in S_{i}(x)$.

According to the generalized Luc's quasi- $C_{i}$-convexity of $F_{i}\left(\cdot, x^{i}\right)$, we get that, for all $z_{i}^{\prime} \in F_{i}\left(\lambda u_{i, 1}+(1-\lambda) u_{i, 2}, x^{i}\right)$, there exist $z_{i, 1} \in F_{i}\left(u_{i, 1}, x^{i}\right)$ and $z_{i, 2} \in F_{i}\left(u_{i, 2}, x^{i}\right)$ such that

$$
z_{i}^{\prime} \in z_{i}-C_{i}, \quad \forall z_{i} \in C\left(z_{i, 1}, z_{i, 2}\right) .
$$

Without loss of generality, suppose $l_{1}=\xi_{e_{i}}\left(z_{i, 1}\right)$ and $l_{2}=$ $\xi_{e_{i}}\left(z_{i, 2}\right), l_{1} \geq l_{2}$; we have $z_{i, 1} \in l_{1} e_{i}-C_{i}$ and $z_{i, 2} \in l_{2} e_{i}-C_{i} \subset$ $l_{1} e_{i}-C_{i}$. From (20), $z_{i}^{\prime} \in l_{1} e_{i}-C_{i}$. By the monotonicity of $\xi_{e_{i}}(\cdot)$,

$$
\xi_{e_{i}}\left(z_{i}^{\prime}\right) \leq \xi_{e_{i}}\left(l_{1} e_{i}\right)=l_{1}
$$

As

$$
l_{1} \leq \max \left(\max \xi_{e_{i}}\left(F\left(u_{i, 1}, x^{i}\right)\right), \max \xi_{e_{i}}\left(F\left(u_{i, 2}, x^{i}\right)\right)\right)=\alpha_{0} .
$$

therefore, $\xi_{e_{i}}\left(z_{i}^{\prime}\right) \leq \alpha_{0}$.

Since

$$
z_{i}^{\prime} \in F_{i}\left(\lambda u_{i, 1}+(1-\lambda) u_{i, 2}, x^{i}\right)
$$

is arbitrary, we have

$$
\max \xi_{e_{i}}\left(F_{i}\left(\lambda u_{i, 1}+(1-\lambda) u_{i, 2}, x^{i}\right)\right) \leq \alpha_{0}
$$

By the fact that $F_{i}\left(\lambda u_{i, 1}+(1-\lambda) u_{i, 2}, x^{i}\right)$ is compact and $\xi_{e_{i}}(\cdot)$ is continuous, there exists

$$
\widetilde{z}_{i} \in F_{i}\left(\lambda u_{i, 1}+(1-\lambda) u_{i, 1}, x^{i}\right)
$$

such that

$$
\xi_{e_{i}}\left(\widetilde{z}_{i}\right)=\max \xi_{e_{i}}\left(F_{i}\left(\lambda u_{i, 1}+(1-\lambda) u_{i, 2}, x^{i}\right)\right) .
$$

Thus, $\xi_{e_{i}}\left(\widetilde{z}_{i}\right) \leq \alpha_{0}$. It follows from the definition of $\alpha_{0}$ that

$$
\max \xi_{e_{i}}\left(F_{i}\left(\lambda u_{i, 1}+(1-\lambda) u_{i, 2}, x^{i}\right)\right)=\alpha_{0}
$$

Thus, $\lambda u_{i, 1}+(1-\lambda) u_{i, 2} \in A_{i}(x)$; namely, $A_{i}(x)$ is a convex set.

Define $A: X \rightarrow 2^{X}$ by $A(x)=\prod_{i \in I} A_{i}(x), \forall x \in X$. Therefore, $A(x)$ is a nonempty, convex, and closed subset of $X$ for each $x \in X$. Since $A_{i}(\cdot)$ is closed, so is $A(\cdot)$, and since $A(x) \subseteq X, X$ is compact, by [12, page 111, Corollary 9], $A(\cdot)$ is upper semicontinuous. By Lemma 5 , there exists a point $\bar{x} \in X$ such that $\bar{x} \in A(\bar{x})$. 
By the definition of $A(\cdot)$, we have

$$
\begin{gathered}
\bar{x}_{i} \in S_{i}(\bar{x}), \\
\max \xi_{e_{i}}\left(F_{i}\left(x_{i}, \bar{x}^{i}\right)\right) \geq \max \xi_{e_{i}}\left(F\left(\bar{x}_{i}, \bar{x}^{i}\right)\right) \\
\forall x_{i} \in S_{i}(\bar{x}), \quad i \in I .
\end{gathered}
$$

From (28), $\forall \bar{z}_{i} \in F_{i}\left(\bar{x}_{i}, \bar{x}^{i}\right)$,

$$
\max \xi_{e_{i}}\left(F_{i}\left(x_{i}, \bar{x}^{i}\right)\right) \geq \xi_{e_{i}}\left(\bar{z}_{i}\right) .
$$

By the compactness of $F_{i}\left(x_{i}, \bar{x}^{i}\right)$ and the continuity of $\xi_{e_{i}}(\cdot)$, there exists $z_{i} \in F_{i}\left(x_{i}, \bar{x}^{i}\right)$, such that $\xi_{e_{i}}\left(z_{i}\right)=$ $\max \xi_{e_{i}}\left(F_{i}\left(x_{i}, \bar{x}^{i}\right)\right)$. Thus, for all $\bar{z}_{i} \in F\left(\bar{x}_{i}, \bar{x}^{i}\right)$, there exists $z_{i} \in F_{i}\left(x_{i}, \bar{x}^{i}\right)$ such that $\xi_{e_{i}}\left(\bar{z}_{i}\right) \leq \xi_{e_{i}}\left(z_{i}\right)$. Then, it follows from the subadditivity of $\xi_{e_{i}}(\cdot)$ that

$$
\xi_{e_{i}}\left(z_{i}-\bar{z}_{i}\right) \geq 0
$$

By Lemma 4, we get

$$
z_{i}-\bar{z}_{i} \notin-\operatorname{int} P
$$

So $\bar{x}$ is a solution of (CWNEP) and this completes the proof.

Let $X, Y$, and $Z$ be real Hausdorff topological vector spaces, and let $C$ and $D$ be two compact subsets of $X$ and $Y$, respectively.

Corollary 7. Let $X, Y$, and $Z$ be real Hausdorff topological vector spaces, and let $C$ and $D$ be two nonempty subsets of $X$ and $Y$, respectively. Let $P \subset Z$ be a closed convex and pointed cone with int $P \neq \emptyset$. Assume that

(1) $S: C \times D \rightarrow 2^{C}$ and $T: C \times D \rightarrow 2^{D}$ are continuous and compact, and for each $(x, y) \in C \times D, S(x, y)$ and $T(x, y)$ are nonempty, closed convex subsets;

(2) $f, g: C \times D \rightarrow Z$ are continuous;

(3) for any fixed $y \in D, f(\cdot, y)$ is Luc's quasi-P-convex; for any fixed $x \in C, g(x, \cdot)$ is Luc's quasi-P-convex.

Then there exists $(\bar{x}, \bar{y}) \in C \times D$ such that $\bar{x} \in S(\bar{x}, \bar{y}), \bar{y} \in$ $T(\bar{x}, \bar{y})$, and

$$
\begin{aligned}
& f(x, \bar{y})-f(\bar{x}, \bar{y}) \notin-\operatorname{int} P, \quad \forall x \in S(\bar{x}, \bar{y}), \\
& g(\bar{x}, y)-g(\bar{x}, \bar{y}) \notin-\operatorname{int} P, \quad \forall y \in T(\bar{x}, \bar{y}) .
\end{aligned}
$$

Remark 8 . Since both the class of properly quasi-P-convex functions and the class of $P$-convex functions (see [7]) are larger than the class of Luc's quasi- $P$-convex functions, Corollary 7 improves [7, Theorem].

Example 9. Suppose that $X=Y=R, C=D=[0,1]$, and $P=R_{+}^{3}$ and let $S: C \times D \rightarrow 2^{C}$ and $T: C \times D \rightarrow 2^{D}$ be defined as $S(x, y)=C$ and $T(x, y)=D$, respectively. For all $(x, y) \in R^{2}$, let

$$
\begin{aligned}
& f(x, y)=\left(x^{2}, 1-x^{2}, y\right), \\
& g(x, y)=\left(x, y^{2}, 1-y^{2}\right) .
\end{aligned}
$$

It is clear that the mappings $f$ and $g$ are not properly quasi- $P$ convex (see [7]), but all the conditions of Corollary 7 hold. It is easy to see from [7] that both the class of properly quasi- $P$ convex functions and the class of $P$-convex functions (see [7]) are larger than the class of Luc's quasi- $P$-convex functions, and then Corollary 7 improves [7, Theorem].

\section{Conflict of Interests}

The authors declare that there is no conflict of interests regarding the publication of this paper.

\section{Acknowledgment}

This paper is supported by the Fundamental Research Funds for the Central Universities (JBK130401 and JBK140924).

\section{References}

[1] D. Blackwell, "An analog of the minimax theorem for vector payoffs," Pacific Journal of Mathematics, vol. 6, pp. 1-8, 1956.

[2] D. Ghose and U. R. Prasad, "Solution concepts in two-person multicriteria games," Journal of Optimization Theory and Applications, vol. 63, no. 2, pp. 167-188, 1989.

[3] F. R. Fernández, M. A. Hinojosa, and J. Puerto, "Core solutions in vector-valued games," Journal of Optimization Theory and Applications, vol. 112, no. 2, pp. 331-360, 2002.

[4] H. W. Corley, "Games with vector payoffs," Journal of Optimization Theory and Applications, vol. 47, no. 4, pp. 491-498, 1985.

[5] L.-J. Lin and S. F. Cheng, "Nash-type equilibrium theorems and competitive Nash-type equilibrium theorems," Computers and Mathematics with Applications, vol. 44, no. 10-11, pp. 1369-1378, 2002.

[6] J. Nash, "Non-cooperative games," The Annals of Mathematics, vol. 54, pp. 286-295, 1951.

[7] J.-Y. Fu, "Symmetric vector quasi-equilibrium problems," Journal of Mathematical Analysis and Applications, vol. 285, no. 2, pp. 708-713, 2003.

[8] T. Tanaka, "Generalized quasiconvexities, cone saddle points, and minimax theorem for vector-valued functions," Journal of Optimization Theory and Applications, vol. 81, no. 2, pp. 355-377, 1994.

[9] C. Gerth and P. Weidner, "Nonconvex separation theorems and some applications in vector optimization," Journal of Optimization Theory and Applications, vol. 67, no. 2, pp. 297320, 1990.

[10] D. T. Luc, Theory of Vector Optimization, Springer, Berlin, Germany, 1989.

[11] V. I. Istratescu, Fixed Point Theory, An Introduction, Direidel, Dordrecht, The Netherlands, 1981.

[12] J. P. Aubin and I. Ekeland, Applied Nonlinear Analysis, John Wiley \& Sons, New York, NY, USA, 1984. 


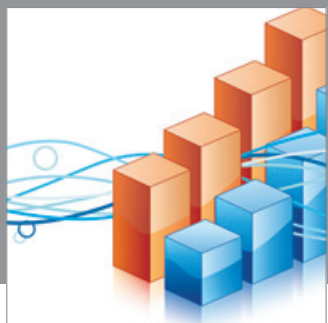

Advances in

Operations Research

mansans

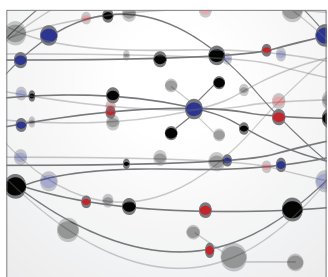

The Scientific World Journal
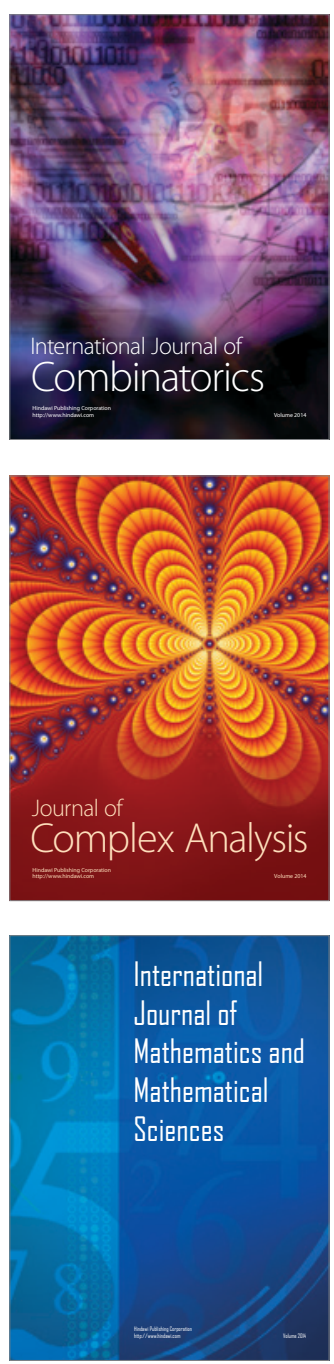
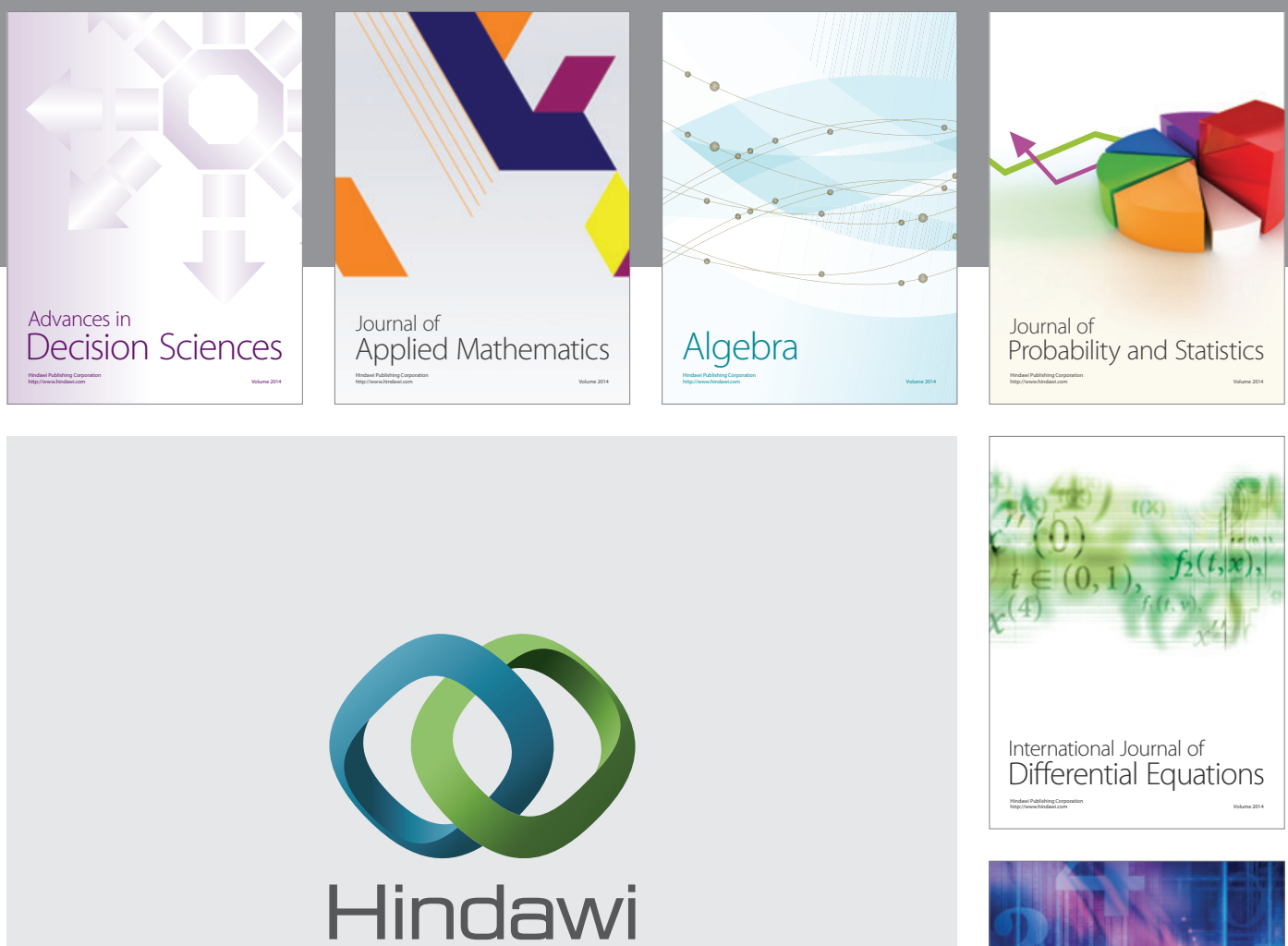

Submit your manuscripts at http://www.hindawi.com
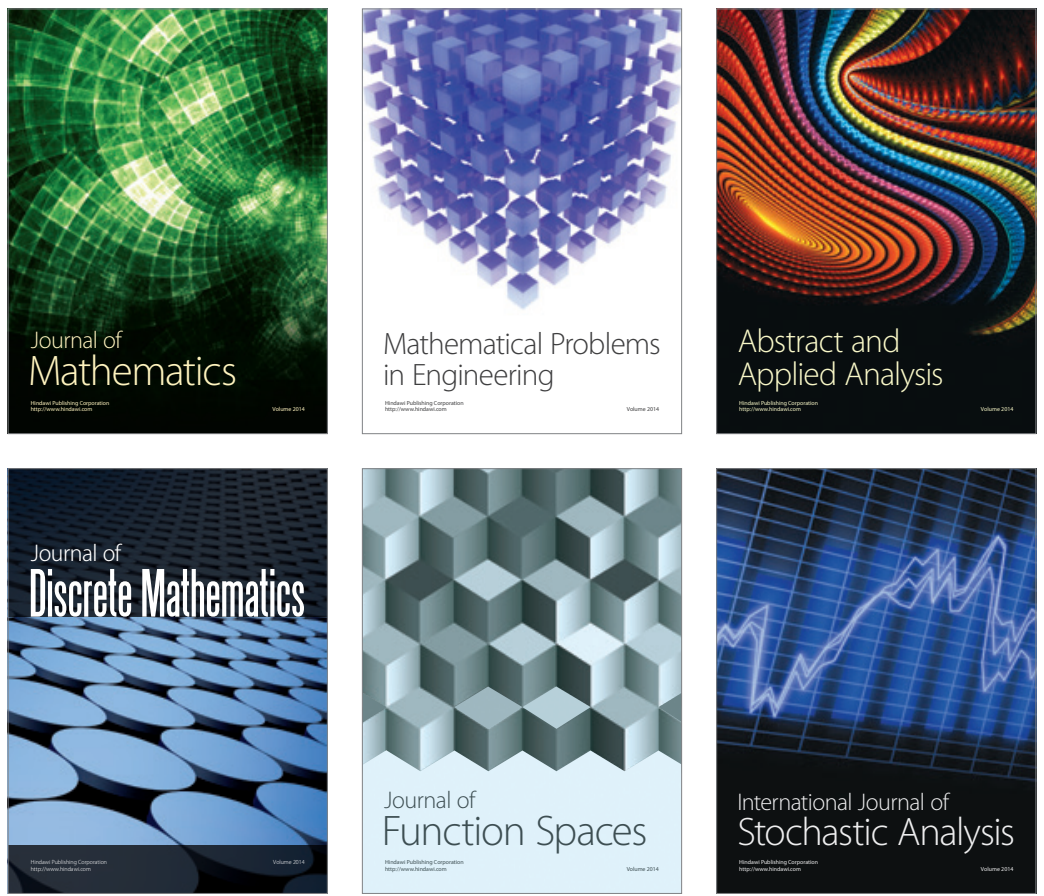

Journal of

Function Spaces

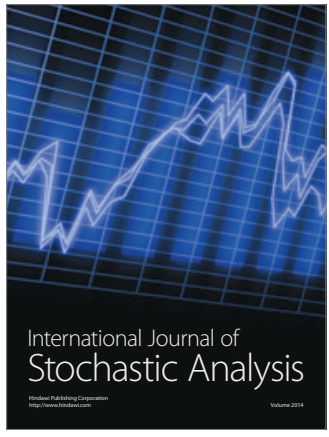

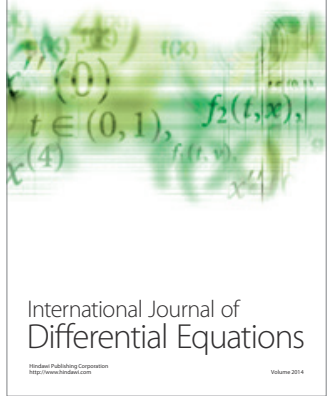
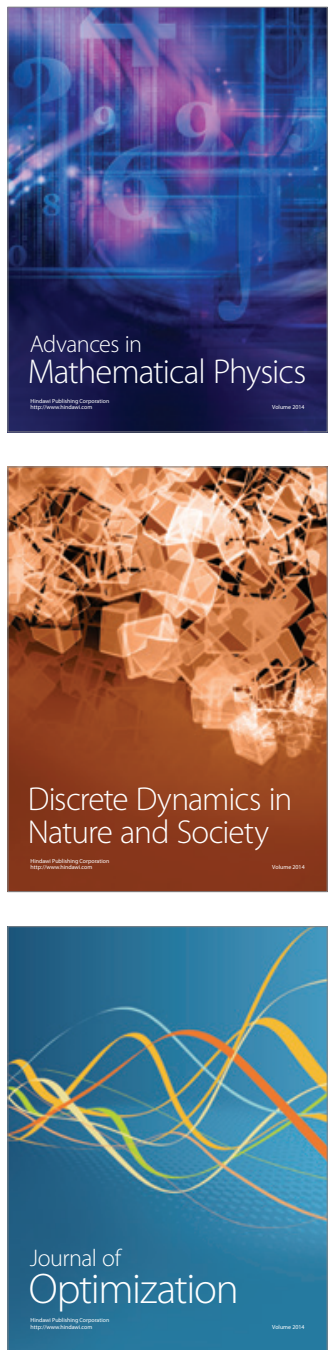